\title{
Metastatic Tumor in the Colon from Renal Cell Carcinoma
}

\author{
Yuichi Nozaki ${ }^{1}$, Masahiko Inamori ${ }^{1}$, Koji Fujita ${ }^{1}$, Masato Yoneda ${ }^{1}$, Shingo Kato ${ }^{1}$, \\ Takashi Uchiyama ${ }^{1}$, Kaori Suzuki ${ }^{1}$, Seitaro Watanabe ${ }^{1}$, Hironori Mawatari ${ }^{1}$, Hiroshi Iida ${ }^{1}$, \\ Kunihiro Hosono ${ }^{1}$, Hiroki Endo ${ }^{1}$, Yasunari Sakamoto ${ }^{1}$, Kyoko Yoneda ${ }^{1}$, \\ Hirokazu Takahashi ${ }^{1}$, Tomoko Koide ${ }^{1}$, Chikako Tokoro ${ }^{1}$, Noritoshi Kobayashi ${ }^{1}$, \\ Hiroyuki Kirikoshi ${ }^{1}$, Takeshi Shimamura ${ }^{1}$, Yasunobu Abe ${ }^{1}$, Kensuke Kubota ${ }^{1}$, \\ Satoru Saito ${ }^{1}$, Hisashi Oshiro ${ }^{2}$, Yoshiaki Inayama ${ }^{2}$ and Atsushi Nakajima ${ }^{1}$
}

Key words: colon tumor, metastasis, renal cell carcinoma

(Inter Med 49: 709, 2010)

(DOI: 10.2169/internalmedicine.49.3233)

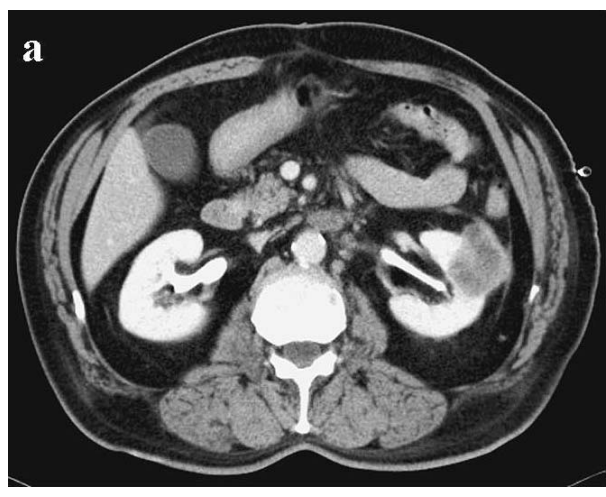

Picture a. Computed tomography revealed a left renal tumor $(35 \mathrm{~mm}$ in diameter) with enhancement.

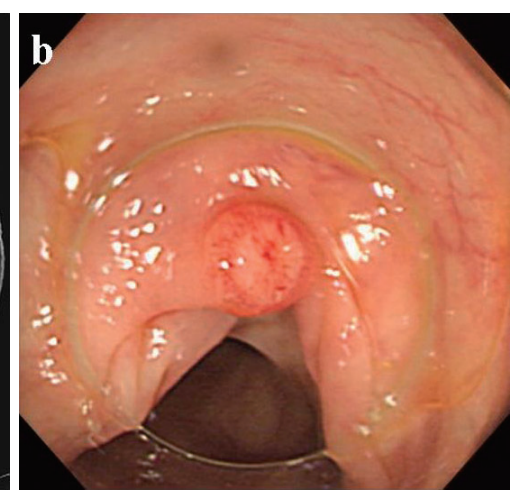

Picture b. Colonoscopy revealed only a submucosal tumor-like tumor measuring $4 \mathrm{~mm}$ in diameter in the transverse colon.

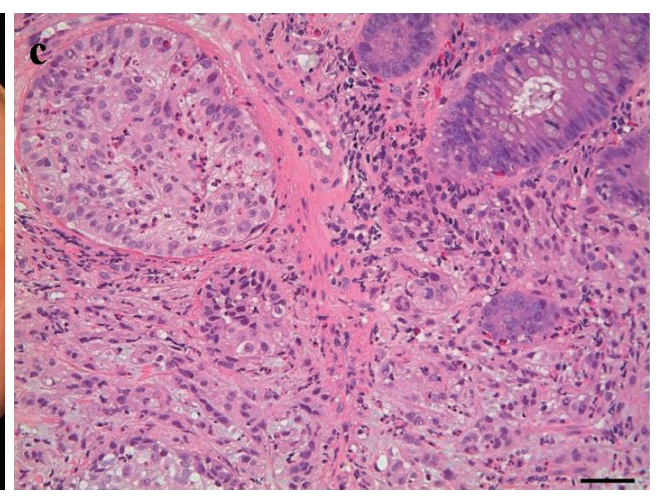

Picture c. Photomicrograph of the colonic biopsy specimen showed metastatic renal cell carcinoma infiltrating colonic mucosal and submucosal layers (Hematoxylin and Eosin staining, bar indicating 50 micrometers).
A 74-year-old man was admitted to our hospital with dyspnea. A chest X-ray showed multiple lesions in both lung fields and left pleural effusion. Computed tomography revealed a left renal tumor $35 \mathrm{~mm}$ in diameter with enhancement (Picture a) and multiple metastatic lesions of both lungs and bones. Colonoscopy revealed only a submucosal tumor-like tumor measuring $4 \mathrm{~mm}$ in diameter in the transverse colon (Picture b). Examination of biopsy speci- mens from the tumor revealed features characteristic of renal cell carcinoma (RCC) (Picture c). Therefore, the patient was diagnosed as having RCC with metastasis to the colon.

There have been few reports of metastatic colon tumors from RCCs. In the present case, we detected a rare metastatic tumor in the colon arising from RCC. The pathway of metastasis to the colon is still not well known.

\footnotetext{
${ }^{1}$ Division of Gastroenterology, Yokohama City University Graduate School of Medicine, Yokohama and ${ }^{2}$ Department of Pathology, Yokohama City University Hospital, Yokohama

Received for publication December 8, 2009; Accepted for publication December 23, 2009

Correspondence to Dr. Masahiko Inamori, inamorim@med.yokohama-cu.ac.jp

(C) 2010 The Japanese Society of Internal Medicine Journal Website: http://www.naika.or.jp/imindex.html
} 